\title{
Impact on disease-free survival of adjuvant erlotinib or gefitinib in patients with resected lung adenocarcinomas that harbor epidermal growth factor receptor (EGFR) mutations
}

\author{
Yelena Y. Janjigian ${ }^{1}$, Bernard J. Park ${ }^{2}$, Maureen F. Zakowski ${ }^{3}$, Marc Ladanyi ${ }^{3}$, William \\ $\mathrm{Pao}^{4}$, Sandra P. D'Angelo ${ }^{5}$, Mark G. Kris ${ }^{5}$, Ronglai Shen ${ }^{6}$, Junting Zheng ${ }^{6}$, and Christopher \\ G. Azzoli ${ }^{5}$ \\ ${ }^{1}$ Gastrointestinal, Memorial Sloan-Kettering Cancer Center, Weill Medical College of Cornell \\ University, New York, NY \\ ${ }^{2}$ Department of Surgery, Memorial Sloan-Kettering Cancer Center, Weill Medical College of \\ Cornell University, New York, NY \\ ${ }^{3}$ Department of Pathology, Memorial Sloan-Kettering Cancer Center, Weill Medical College of \\ Cornell University, New York, NY \\ ${ }^{4}$ Vanderbilt-Ingram Cancer Center, Vanderbilt University,Nashville, TN \\ ${ }^{5}$ Thoracic Oncology Services, Division of Solid Tumor Oncology, Department of Medicine, \\ Memorial Sloan-Kettering Cancer Center, Weill Medical College of Cornell University, New York, \\ NY \\ ${ }^{6}$ Department of Epidemiology and Biostatistics, Memorial Sloan-Kettering Cancer Center, Weill \\ Medical College of Cornell University, New York, NY
}

\section{Abstract}

Background-Patients with stage IV lung adenocarcinoma and EGFR mutation derive clinical benefit from treatment with EGFR tyrosine kinase inhibitors (TKI). Whether treatment with TKI improves outcomes in patients with resected lung adenocarcinoma and EGFR mutation is unknown.

\begin{abstract}
Methods-Data were analyzed from a surgical database of patients with resected lung adenocarcinoma harboring EGFR exon 19 or 21 mutations. In a multivariate analysis, we evaluated the impact of treatment with adjuvant TKI.
\end{abstract}

\begin{abstract}
Results-The cohort consists of 167 patients with completely resected stage I-III lung adenocarcinoma. 93 patients (56\%) had exon 19 del, 74 patients (44\%) had exon 21 mutations, 56 patients $(33 \%)$ received perioperative TKI. In a multivariate analysis controlling for sex, stage, type of surgery and adjuvant platinum chemotherapy, the 2-year DFS was $89 \%$ for patients treated with adjuvant TKI compared with $72 \%$ in control group (hazard ratio $[\mathrm{HR}]=0.53 ; 95 \%$ confidence interval [CI] 0.28 to $1.03 ; p=0.06$ ). The 2 -year OS was $96 \%$ with adjuvant EGFR TKI and $90 \%$ in the group that did not receive TKI (HR $0.62 ; 95 \%$ CI 0.26 to $1.51 ; p=0.296$ ).
\end{abstract}

Conclusions-Compared to patients who did not receive adjuvant TKI, we observed a trend toward improvement in disease free survival among individuals with resected stages I-III lung adenocarcinomas harboring mutations in EGFR exons 19 or 21 who received these agents as

Address Correspondence to: Christopher Azzoli, MD, Department of Medicine, Memorial Sloan-Kettering Cancer Center, 1275 York Avenue, New York, NY 10065, T - 212-639-2131, F - 212-794-4357, azzolic@ mskcc.org.

Presented in part at the ASCO Annual Meeting, Orlando, Florida May 30-June 3, 2009. 
adjuvant therapy. Based on these data, 320 patients are needed for a randomized trial to prospectively validate this DFS benefit.

\section{Introduction}

The most effective treatment for lung adenocarcinoma is complete (R0) surgical resection, with anatomical resection (lobectomy or pneumonectomy) as the preferred surgical procedure. ${ }^{1}$ However, even for patients who have complete resections, many are not cured, and rates of death 5 years after resection vary from $30 \%$ in stage IA to $75 \%$ in stage IIIA. ${ }^{2}$ Perioperative cisplatin-based chemotherapy given before and/or after surgery can increase overall survival after NSCLC resection by treating occult metastatic disease. ${ }^{3-6}$

Perioperative cytotoxic chemotherapy reduces the risk of death at 5 years by approximately $15 \%$ (absolute risk reduction) in patients with stage IIIA disease, and approximately $4 \%$ for patients with stage IB NSCLC. For stage IA disease, despite the unacceptably high recurrence rate at 5 years, treatment with adjuvant cisplatin chemotherapy has no survival benefit. $^{7}$

NSCLC is a heterogeneous disease, with activating mutations in epidermal growth factor receptor $(E G F R)$ playing a key role in the growth and proliferation in about $20 \%$ of lung adenocarcinomas. Multiple prospective trials demonstrate that individuals with advanced lung adenocarcinomas that harbor EGFR mutations, deletions in exon 19, or L858R missense mutations at exon 21, experience a high rate of radiologic response with the EGFR tyrosine kinase inhibitors (TKIs) erlotinib (Tarceva) or gefitinib (Iressa). ${ }^{8-11}$

Three randomized phase 3 trials demonstrated that gefitinib is superior to platinum-based chemotherapy as an initial treatment for advanced lung adenocarcinoma among patients with $E G F R$ mutations, improving both radiologic response and progression-free survival. ${ }^{12-14}$ The presence of EGFR mutations may be an independent, good prognostic factor in lung adenocarcinoma irrespective of therapy. ${ }^{12,15-17}$

Since EGFR TKIs are superior to cytotoxic drugs in patients with advanced lung adenocarcinoma and EGFR mutation, many have proposed that EGFR TKIs may also prove advantageous when used as adjuvant treatments in this population. As such, our institution routinely tests all resected lung adenocarcinomas for the presence of EGFR mutations. This has allowed our participation in ongoing, prospective clinical trials of EGFR TKIs for patients with EGFR mutations (NCT00567359). It also affords the opportunity to observe the clinical outcome of patients who received adjuvant EGFR TKIs outside of a clinical trial. To accomplish this, we reviewed a prospectively-maintained surgical database which includes clinical and molecular characteristics and outcome data for patients with resected stage I-III lung adenocarcinoma with EGFR mutations.

\section{Patients and Methods}

\section{Patients}

In an Institutional Review Board approved surgical database we identified all patients with completely resected stage I-III lung adenocarcinomas with EGFR mutations who had a resection at Memorial Sloan-Kettering Cancer Center (MSKCC) between May 2002 and August 2008. Some of these patients received EGFR tyrosine kinase inhibitors (TKIs) gefitinib or erlotinib pre- and/or postoperatively as routine care, or under the auspices of a clinical trial of perioperative gefitinib. ${ }^{18}$ The surgical database prospectively captures patient sex, age, type of surgery, histology, pathologic stage, perioperative chemotherapy and radiation. From the medical record, we obtained the information regarding erlotinib or gefitinib delivery, disease status and survival. This review of records was done under a 
waiver of authorization approved by the MSKCC Institutional Review Board and Privacy Board. According to the consensus of MSKCC surgeons and medical oncologists, patients at MSKCC are followed for disease recurrence in uniform fashion, including a doctor visit and CT scan of the chest (preferably with IV contrast) every 6 months until the two-year anniversary of the date of surgery, and non-contrast CT annually thereafter.

\section{EGFR mutation testing}

Since January, 2006, EGFR mutation testing has been performed reflexively (automatically) at MSKCC by the molecular diagnostic core laboratory of the Department of Pathology on all resected lung adenocarcinomas. DNA is extracted from formalin-fixed, paraffinembedded tumor tissue and tested by a polymerase chain reaction-based assays for the two predominant types of EGFR mutations- short in-frame deletions in exon 19 and a specific point mutation in exon 21 at codon 858 (L858R) (methods described in ${ }^{19}$ ). Prior to 2006, mutation testing was done on many, although not all resected patients using a variety of techniques, including direct sequencing. Using direct sequencing, tumors from 2 patients were found to have a point mutation in exon 21 at codon 861 (L861Q), which is also associated with sensitivity to erlotinib and gefitinib. ${ }^{20-22}$

\section{Statistical Analysis}

The characteristics of patients who received neoadjuvant and/or adjuvant TKI were summarized and compared to the group that did not receive TKI using Wilcoxon rank-sum test or Fisher's exact test. Disease free survival (DFS) was measured from date of surgery to date of recurrence or death. Overall survival time (OS) was measured from date of surgery to date of death. Living patients were censored at the date of last contact. Survival data were obtained using the medical record and the Social Security Death Index. Survival status was updated in March 2010. Survival probabilities were calculated by the Kaplan-Meier method and compared among different groups using the log-rank test. Univariate and multivariate Cox regression analyses were performed controlling for sex, stage, surgical procedure and perioperative platinum-based chemotherapy. Perioperative erlotinib or gefitinib therapy was treated as a time dependent factor so that when it was given after the surgery, its effect would not be taken into account until the start of the therapy. Statistical analyses were done using SAS (SAS Institute, Inc., Cary, NC) software.

\section{Results}

\section{Patient Characteristics}

We identified 167 patients with stage I-III completely resected lung adenocarcinomas harboring EGFR exon 19 or 21 mutations that underwent resection at MSKCC between May 2002 and August 2008. The demographic characteristics of the patients are summarized in Table 1 . The cohort consists predominantly (70\%) of stage I resected lung adenocarcinomas such that only 47 patients (28\%) in the entire cohort received perioperative cisplatin-based chemotherapy. Fifty six patients (33\%) received perioperative erlotinib or gefitinib. The adjuvant erlotinib/gefitinib group had a higher proportion of stage III patients. Stage distribution among the group is summarized in Table 1 . Twenty one of the adjuvant TKItreated patients captured by the database were enrolled in a phase 2 clinical trial and received perioperative gefitinib consisting of induction therapy for 21 days prior to surgery and again postoperatively for up to 2 years. ${ }^{18}$ The remaining 35 patients received postoperative erlotinib or gefitinib at the discretion of their physicians. The median time between start of erlotinib/gefitinib therapy and surgery was 2 months. The median time on erlotinib or gefitinib was 20 months (range, 0.3 to 51 months). 


\section{Survival Analysis}

To date, a total of 46 recurrences (27\%) and 29 deaths (17\%) have occurred. Ten patients died without documented disease recurrence. Table 2 summarizes the characteristics of patients who recurred or died. Of the patients who recurred, 43 developed unresectable metastatic disease: 8 brain/leptomeninges only, 15 lung/pleura only and 20 recurrences in multiple disease sites: brain, bone, lung, liver or lymph nodes. Three patients were rendered free of disease recurrence in the lung with surgery. These tumors were all histopathologically similar to the primary resection specimens with identical EGFR mutations, excluding a second primary lung tumor as a possibility.

In the entire cohort of completely resected stage I-III lung adenocarcinoma harboring EGFR mutations, the median OS and DFS of all patients were 76 months (95\% CI, 71 to NA) and 46 months (95\% CI, 40 to 67) respectively. Kaplan-Meier survival estimates are provided in Figure 1. In a multivariate analysis, after adjusting for sex, pathologic stage, surgery type and application of cisplatin-based chemotherapy, patients treated with perioperative erlotinib/gefitinib therapy had a 2-year disease free survival rate of $89 \%$ compared to $72 \%$ for patients that were not treated with perioperative erlotinib/gefitinib $(p=0.06)$, shown in Table 3. Of the 46 patients that experienced disease recurrence, 32 (69\%) received EGFR tyrosine kinase inhibitor therapy for recurrent disease, 1 patient was rendered disease free with resection and is being monitored off therapy, 3 patients died without receiving erlotinib or gefitinib; and for 10 patients the therapy for metastatic disease is unknown. In a multivariate analysis, patients treated with perioperative erlotinib/gefitinib had a 2-year overall survival of $96 \%$ compared to $90 \%$ for patients that were not treated with perioperative erlotinib/gefitinib $(\mathrm{p}=0.296)$ shown in Table 4 .

\section{Discussion}

In advanced lung adenocarcinoma, the response rates to EGFR TKI therapy in patients with EGFR mutations range from $58 \%$ to $75 \%$ in prospective trials. ${ }^{9-12,15,23-25}$ Three randomized phase 3 studies have demonstrated that EGFR TKI therapy is superior to chemotherapy in this unique subset of advanced NSCLC patients. ${ }^{12-14}$ If we assume that patients with micrometastatic disease after surgery would have a similar benefit, we can hypothesize that adjuvant EGFR TKI could delay relapse or potentially cure patients with resectable cancer who would otherwise relapse with adjuvant cisplatin-based chemotherapy alone. In addition to being potentially more effective than adjuvant cisplatin-based chemotherapy in EGFR mutation-positive patients, EGFR TKIs have fewer side effects making them an appealing treatment option for patients with stage IA NSCLC, a subgroup for which no adjuvant therapy currently exists. ${ }^{7}$

An important example of molecularly-targeted adjuvant therapy for patients with resected solid tumors is imatinib mesylate (Gleevec) therapy for patients with gastrointestinal stromal tumors (GIST) which express KIT protein (CD117) by immunohistochemistry. ${ }^{26-28}$ A 770 patient randomized, placebo-controlled phase 3 trial demonstrated an improvement in disease free survival for the imatinib mesylate arm (HR 0.35; 95\% CI, 0.22 to 0.53 , $\mathrm{p}<0.0001) .{ }^{28}$ To date, an improvement in overall survival from adjuvant imatinib has not been established. Studies are ongoing to determine whether the decision to treat, or dose of imatinib, should vary based on the presence of KIT gene mutations.

As for adjuvant use of EGFR TKI for patients with resected EGFR mutant lung adenocarcinoma, there are no randomized trials directing this therapy. A multi-center, single-arm, phase 2 trial (NCT00567359) is underway at Massachusetts General Hospital (MGH), MGH affiliated hospitals, and MSKCC which will prospectively enroll 100 patients with resected NSCLC and activating mutations in EGFR to receive erlotinib $150 \mathrm{mg}$ daily 
dose for up to two years after completion of all standard adjuvant chemotherapy and radiation therapy. ${ }^{30}$ Thirty six patients have been enrolled over a course of two years. Rash, fatigue and diarrhea were the most common grade 3 toxicities occurring in 14\%, 3\% and $6 \%$ of patients respectively. Ten (28\%) patients were dose reduced to erlotinib $100 \mathrm{mg}(\mathrm{n}=6)$ and $50 \mathrm{mg}(\mathrm{n}=4)$ due to toxicities and $5(14 \%)$ have withdrawn as a result of toxicity or patient preference. ${ }^{30}$ The primary endpoint is DFS at 2 years. Since this is a single arm trial, the data for patients with resected stage I-III NSCLC and EGFR mutation who do not receive EGFR TKI being collected at MSKCC will serve as an important historical comparator.

Two randomized trials of adjuvant EGFR TKI for patients with resected NSLC have been initiated, but neither trial is enriched for patients with EGFR mutations. There is consensus that the greatest benefit of EGFR TKI therapy occurs in metastatic patients with EGFR mutations ${ }^{12}$ and that there is growing concern that there may be harm to EGFR wild type or $K R A S$ mutant patients treated with erlotinib or gefitinib ${ }^{12}$. Any data from EGFR TKI trials that do not select patients based on presence of EGFR mutations may be misleading.

The phase 3 National Cancer Institute of Canada BR19 trial comparing adjuvant gefitinib versus placebo in patients with completely resected NSCLC was initiated, but closed early for safety concerns after interim analysis of phase 3 S0023 trial showed that maintenance gefitinib was worse than placebo in unselected patients with inoperable stage III NSCLC. ${ }^{31}$ As a result, BR19 accrued 503 of planned 1160 patients with median duration of active study therapy of less than 5 months. The study eligibility criteria did not mandate selection of patients based on molecular or clinical characteristics that correlate with sensitivity to gefitinib such as adenocarcinoma histology, female gender, never smoking status, or East Asian ethnicity..$^{32,33}$ The majority of patients enrolled in BR19 were former or current smokers, and included patients with squamous cell histology. ${ }^{34}$ Tissue samples from 357 of 503 patients who received treatment on BR19 were studied with the goal of demonstrating a clinical benefit for adjuvant gefitinib in the subgroup of patients with EGFR mutations. Mutation status in $30 \%$ of patients treated on BR19 remains unknown. Since the BR19 patient population was not enriched for presence of $E G F R$ mutations and mutation testing was limited to a subgroup of patients, only 76 patients with EGFR mutations (40 in the placebo arm and 36 in the gefitinib arm) were included in the study. ${ }^{34}$ The incidence for grade 3 or 4 adverse events was very low, with dyspnea being the most common serious adverse event and equally balanced between gefitinib and placebo arms seen in 5\% of patients in each treatment arm. A higher incidence of pneumonitis was noted in the placebo arm. In exploratory analysis subset of patients with EGFR mutations gefitinib therapy did not result in disease free or overall survival benefit. ${ }^{34}$ Taking into account that this was a subgroup analysis of an underpowered study that was terminated early with some patients receiving only a few days of study therapy, the results of BR19 can not be used to draw conclusions about the impact of adjuvant EGFR TKIs in patients with lung adenocarcinoma and $E G F R$ mutation.

The RADIANT (Randomized Double-Blind Trial In Adjuvant NSCLC with Tarceva) is another phase 3 trial that is currently investigating adjuvant erlotinib therapy in resected NSCLC patients who have overexpression of EGFR protein by immunohistochemistry (IHC) or EGFR gene amplification by fluorescence in situ hybridization (FISH). Although $E G F R$ mutations are not a prerequisite for the RADIANT study entry, EGFR mutation analysis was performed on tumors from 278 patients enrolled to date. As expected, the rate of EGFR exon 19 and 21 mutations in this unselected patient population is $12 \% .{ }^{35}$ The planned accrual for the RADIANT trial is 945 patients, which will result in approximately 113 patients (about 60 patients per treatment arm) with tumors that harbor $E G F R$ mutation. Again, based on our data, it is unlikely that a significant difference in outcomes can be 
demonstrated in this number of patients. Subdividing the data into subgroups reduces the study's power to detect treatment differences by reducing the sample size, and increasing the number of statistical tests needed to test for an interaction between different clinical factors. ${ }^{36}$

In contrast to the RADIANT and BR19 trials, our cohort consists exclusively of patients with resected lung adenocarcinomas with EGFR mutations. The majority of molecular testing on our patients was performed prospectively and all testing was done at a single laboratory. In regard to surgery and chemotherapy, the majority of the patients in this cohort had uniform management for their lung adenocarcinoma by the MSKCC Thoracic Disease Management Team.

While the large number of patients from a single institution makes this data important, there are limitations to our analysis. Treatment with adjuvant erlotinib/gefitinib was largely dependent on the patients' and their oncologists' preference. Seventy percent of patients who received the EGFR TKI had Stage I-II disease and given the long natural history of early stage EGFR mutant lung adenocarcinoma, the 2 year disease free survival may be too short to adequately reflect the population outcome. Patients with more advanced tumors were more likely to be offered, or motivated, to take adjuvant TKI therapy as an innovative therapy after surgery and completion of standard adjuvant therapy. Since more high-risk patients were treated with adjuvant TKI, this would tend to bias the results against the therapeutic impact of the TKI. We accounted for differences in prognostic variables between patients who did, and did not receive adjuvant TKI by adjusting for age, sex, and pathologic stage through a multivariate analysis. A proportion of patients received gefitinib therapy preoperative and postoperatively, while others only received erlotinib postoperatively. Finally, we did not control for the doses or duration of EGFR TKI therapy, and we did not collect toxicity data prospectively, although no patient was identified whose death was attributed to treatment with erlotinib or gefitinib.

Despite the study limitations, we have demonstrated for the first time that adjuvant therapy with erlotinib or gefitinib in patients with resected lung adenocarcinoma harboring EGFR mutations may have an impact on disease free survival (HR 0.53, 95\% CI: 0.28 to $1.03 \mathrm{p}$ $=0.06$ ). Given the potential magnitude of disease free survival benefit our data supports, and the relatively small number of patients needed in a randomized trial to confirm these observations, a phase 3 trial is justified. The primary endpoint should be disease free survival, the same endpoint that was used in the trial that lead to the approval of imatinib in patients with KIT-positive gastrointestinal stromal tumors. ${ }^{26,27}$ Overall survival cannot be used as the primary endpoint because virtually all patients with recurrent lung adenocarcinoma and EGFR mutation will receive an EGFR TKI at recurrence. Based on our results, the number of $E G F R$ mutation-positive lung adenocarcinoma cases in RADIANT (estimate $\mathrm{n}=120)$, BR19 $(\mathrm{n}=76)$, and the ongoing single-arm NCT00567359 trial $(\mathrm{n}=100)$ pooled together will be insufficient to prove a clinically significant disease free survival advantage of adjuvant EGFR TKI therapy. Appropriate patient selection with deliberate trial design and sample size justification will be essential to demonstrate the survival benefit for adjuvant therapy in a resected lung adenocarcinoma population that may have a favorable prognosis irrespective of therapy.

This analysis was performed to provide the framework for further investigation of adjuvant EGFR TKI therapy in lung adenocarcinoma patients with EGFR mutations. Based on this data, we propose a phase 3 study to detect a $47 \%$ reduction in the risk of lung cancer recurrence or death. To demonstrate this improvement in disease free survival, 320 patients would be needed, with 160 patients per treatment arm, over a 3 year accrual time and 2 year follow up (trial design proposed in Figure 2). This design provides $85 \%$ power at $5 \%$ type I 
error rate. There is a theoretical possibility that adjuvant TKI might improve disease free survival, but injure patients or breed resistant clones which, in the long run, might result in worse overall survival (OS). As such, we propose that the validation protocol will measure OS, and mandate that enrolled patients with recurrent cancer must be treated with first-line TKI at recurrence, thus allowing a comparison of radiologic response rate and progression free survival between the two arms of the study at recurrence giving early insight into the potential problem of acquired resistance to EGFR TKIs which may develop from adjuvant TKI therapy.

\section{Acknowledgments}

Supported, in part, by NIH 5T32CA009207

\section{References}

1. Ginsberg RJ, Rubinstein LV. Randomized trial of lobectomy versus limited resection for T1 N0 non-small cell lung cancer. Lung Cancer Study Group. Ann Thorac Surg. 1995; 60:615-22. discussion 622-3. [PubMed: 7677489]

2. Goldstraw P, Crowley J, Chansky K, et al. The IASLC Lung Cancer Staging Project: proposals for the revision of the TNM stage groupings in the forthcoming (seventh) edition of the TNM Classification of malignant tumours. J Thorac Oncol. 2007; 2:706-14. [PubMed: 17762336]

3. Douillard JY, Rosell R, De Lena M, et al. Adjuvant vinorelbine plus cisplatin versus observation in patients with completely resected stage IB-IIIA non-small-cell lung cancer (Adjuvant Navelbine International Trialist Association [ANITA]): a randomised controlled trial. Lancet Oncol. 2006; 7:719-27. [PubMed: 16945766]

4. Winton T, Livingston R, Johnson D, et al. Vinorelbine plus cisplatin vs. observation in resected nonsmall-cell lung cancer. N Engl J Med. 2005; 352:2589-97. [PubMed: 15972865]

5. Arriagada R, Bergman B, Dunant A, et al. Cisplatin-based adjuvant chemotherapy in patients with completely resected non-small-cell lung cancer. N Engl J Med. 2004; 350:351-60. [PubMed: 14736927]

6. Kato H, Ichinose Y, Ohta M, et al. A randomized trial of adjuvant chemotherapy with uracil-tegafur for adenocarcinoma of the lung. N Engl J Med. 2004; 350:1713-21. [PubMed: 15102997]

7. Pignon J-P, Tribodet H, Scagliotti GV, et al. Lung Adjuvant Cisplatin Evaluation: A Pooled Analysis by the LACE Collaborative Group. J Clin Oncol. 2008; 26:3552-3559. [PubMed: 18506026]

8. Paz-Ares L, Sanchez JM, García-Velasco A, et al. A prospective phase II trial of erlotinib in advanced non-small cell lung cancer (NSCLC) patients (p) with mutations in the tyrosine kinase (TK) domain of the epidermal growth factor receptor (EGFR). Journal of Clinical Oncology, 2006 ASCO Annual Meeting Proceedings Part I. 2006; 24:7020.

9. Pignon JP, Tribodet H, Scagliotti GV, et al. Lung Adjuvant Cisplatin Evaluation (LACE): A pooled analysis of five randomized clinical trials including 4,584 patients. J Clin Oncol (Meeting Abstracts). 2006; 24:7008.

10. Sunaga N, Yanagitani N, Kaira K, Tomizawa Y, Iijima H, Otani Y, Tanaka S, Suga T, Dobashi K, Mori M. Phase II study of the efficacy of gefitinib in patients with non-small cell lung cancer with the EGFR mutations. J Clin Oncol. 2006; 24

11. Sutani A, Nagai Y, Udagawa K, et al. Phase II study of gefitinib for non-small cell lung cancer (NSCLC) patients with epidermal growth factor receptor (EGFR) gene mutations detected by PNA-LNA PCR clamp. J Clin Oncol (Meeting Abstracts). 2006; 24:7076.

12. Mok TS, Wu Y-L, Thongprasert S, et al. Gefitinib or Carboplatin-Paclitaxel in Pulmonary Adenocarcinoma. N Engl J Med. 2009; 361:947-957. [PubMed: 19692680]

13. Maemondo M, Inoue A, Kobayashi K, et al. Gefitinib or Chemotherapy for Non-Small-Cell Lung Cancer with Mutated EGFR. N Engl J Med. 2010; 362:2380-2388. [PubMed: 20573926]

14. Mitsudomi T, Morita S, Yatabe Y, et al. Gefitinib versus cisplatin plus docetaxel in patients with non-small-cell lung cancer harbouring mutations of the epidermal growth factor receptor 
(WJTOG3405): an open label, randomised phase 3 trial. Lancet Oncol. 2010; 11:121-8. [PubMed: 20022809]

15. Eberhard DA, Johnson BE, Amler LC, et al. Mutations in the epidermal growth factor receptor and in KRAS are predictive and prognostic indicators in patients with non-small-cell lung cancer treated with chemotherapy alone and in combination with erlotinib. J Clin Oncol. 2005; 23:59009. [PubMed: 16043828]

16. Janjigian, YYPB.; Kris, MG. Prognostic Implications of EGFR and KRAS Gene Mutations in Resected Lung Adenocarcinoma. J Thor Oncology; 13th World Conference on Lung Cancer; San Fracisco, CA. 2009.

17. Liu H-P, Isaac Wu H-D, Chang JW-C, et al. Prognostic Implications of Epidermal Growth Factor Receptor and KRAS Gene Mutations and Epidermal Growth Factor Receptor Gene Copy Numbers in Patients with Surgically Resectable Non-small Cell Lung Cancer in Taiwan. Journal of Thoracic Oncology. 2010; 5:1175-1184.10.1097/JTO.0b013e3181e2f4d6 [PubMed: 20559151]

18. Rizvi NA, Pao W, Kris MG, et al. A prospective study to correlate EGFR mutations with gefitinib response. J Clin Oncol (Meeting Abstracts). 2005; 23:7091.

19. Pan Q, Pao W, Ladanyi M. Rapid Polymerase Chain Reaction-Based Detection of Epidermal Growth Factor Receptor Gene Mutations in Lung Adenocarcinomas. J Mol Diagn. 2005; 7:396403. [PubMed: 16049312]

20. Lynch TJ, Bell DW, Sordella R, et al. Activating mutations in the epidermal growth factor receptor underlying responsiveness of non-small-cell lung cancer to gefitinib. N Engl J Med. 2004; 350:2129-39. [PubMed: 15118073]

21. Paez JG, Janne PA, Lee JC, et al. EGFR Mutations in Lung Cancer: Correlation with Clinical Response to Gefitinib Therapy. Science. 2004; 304:1497-1500. [PubMed: 15118125]

22. Pao W, Miller V, Zakowski M, et al. EGF receptor gene mutations are common in lung cancers from "never smokers" and are associated with sensitivity of tumors to gefitinib and erlotinib. Proc Natl Acad Sci USA. 2004; 101:13306-13311. [PubMed: 15329413]

23. Inoue A, Suzuki T, Fukuhara T, et al. Prospective phase II study of gefitinib for chemotherapynaive patients with advanced non-small-cell lung cancer with epidermal growth factor receptor gene mutations. J Clin Oncol. 2006; 24:3340-6. [PubMed: 16785471]

24. Paz-Ares L, Sanchez JM, Garcia-Velasco A, et al. A prospective phase II trial of erlotinib in advanced non-small cell lung cancer (NSCLC) patients (p) with mutations in the tyrosine kinase (TK) domain of the epidermal growth factor receptor (EGFR). J Clin Oncol (Meeting Abstracts). 2006; $24: 7020$.

25. Sunaga N, Tomizawa Y, Yanagitani N, et al. Phase II prospective study of the efficacy of gefitinib for the treatment of stage III/IV non-small cell lung cancer with EGFR mutations, irrespective of previous chemotherapy. Lung Cancer. 2007; 56:383-9. [PubMed: 17368623]

26. Nilsson B, Sjolund K, Kindblom LG, et al. Adjuvant imatinib treatment improves recurrence-free survival in patients with high-risk gastrointestinal stromal tumours (GIST). Br J Cancer. 2007; 96:1656-1658. [PubMed: 17533389]

27. Cohen MH, Cortazar P, Justice R, et al. Approval Summary: Imatinib Mesylate in the Adjuvant Treatment of Malignant Gastrointestinal Stromal Tumors. Oncologist. 2010; 15:300-307. [PubMed: 20200041]

28. Dematteo RP, Ballman KV, Antonescu CR, et al. Adjuvant imatinib mesylate after resection of localised, primary gastrointestinal stromal tumour: a randomised, double-blind, placebo-controlled trial. Lancet. 2009; 373:1097-104. [PubMed: 19303137]

29. Comparison of two doses of imatinib for the treatment of unresectable or metastatic gastrointestinal stromal tumors. a meta-analysis of 1,640 patients. J Clin Oncol. 2010; 28:1247-53. [PubMed: 20124181]

30. Neal JWNAP, Goodgame BW, Lanuti M, Heist RS, Shaw AT, Temel JS, Janne PA, Azzoli CG, Sequist LV. A multicenter phase II trial of adjuvant erlotinib in patients with resected non-small cell lung cancer (NSCLC) and mutations in the epidermal growth factor receptor (EGFR): Toxicity evaluation. J Clin Oncol. 2010; 28:15s. (suppl; abstr 7078), 2010.

31. Kelly K, Chansky K, Gaspar LE, et al. Updated analysis of SWOG 0023: A randomized phase III trial of gefitinib versus placebo maintenance after definitive chemoradiation followed by docetaxel 
in patients with locally advanced stage III non-small cell lung cancer. Journal of Clinical Oncology, 2007 ASCO Annual Meeting Proceedings Part I. 2007; 25:7513.

32. Riely GJ, Politi KA, Miller VA, et al. Update on Epidermal Growth Factor Receptor Mutations in Non-Small Cell Lung Cancer. Clinical Cancer Research. 2006; 12:7232-7241. [PubMed: 17189394]

33. Marchetti A, Martella C, Felicioni L, et al. EGFR Mutations in Non-Small-Cell Lung Cancer: Analysis of a Large Series of Cases and Development of a Rapid and Sensitive Method for Diagnostic Screening With Potential Implications on Pharmacologic Treatment. J Clin Oncol. 2005; 23:857-865. [PubMed: 15681531]

34. Goss GDIL, Tsao MS, O'Callaghan CJ, Ding K, Masters GA, Gandara DR, Jett JR, Edelman MJ, Shepherd FA. A phase III randomized, double-blind, placebo-controlled trial of the epidermal growth factor receptor inhibitor gefitinb in completely resected stage IB-IIIA non-small cell lung cancer (NSCLC): NCIC CTG BR.19. J Clin Oncol. 2010; 28:18s. (suppl; abstr LBA7005), 2010.

35. Richardson F, Richardson K, Sennello G, Young D, Orlov S, Pápai-Székely Z, Keshavjee S, Kim J, Cerfolio R, Shepherd FA. Biomarker analysis from completely resected NSCLC patients enrolled in an adjuvant erlotinib clinical trial (RADIANT). J Clin Oncol. 2009; 27:15s. (suppl; abstr 7520).

36. Lagakos SW. The Challenge of Subgroup Analyses: Reporting without Distorting. New England Journal of Medicine. 2006; 354:1667-1669. [PubMed: 16625007] 
A

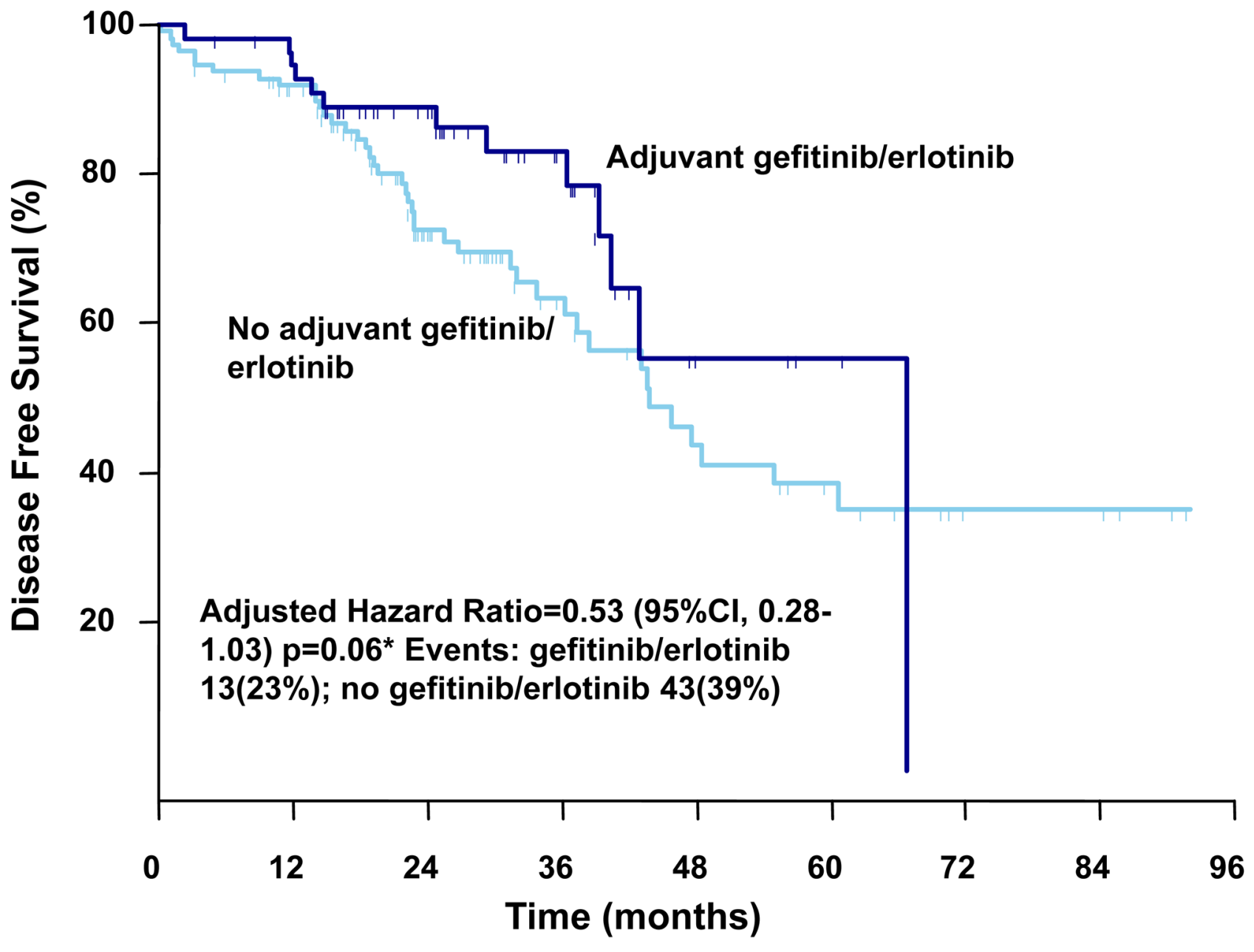

Number at Risk

no G/E therapy $\mathbf{9 5}$

52

28

17

11

6

5

G/E therapy 51

37

18

5

2

0

0 


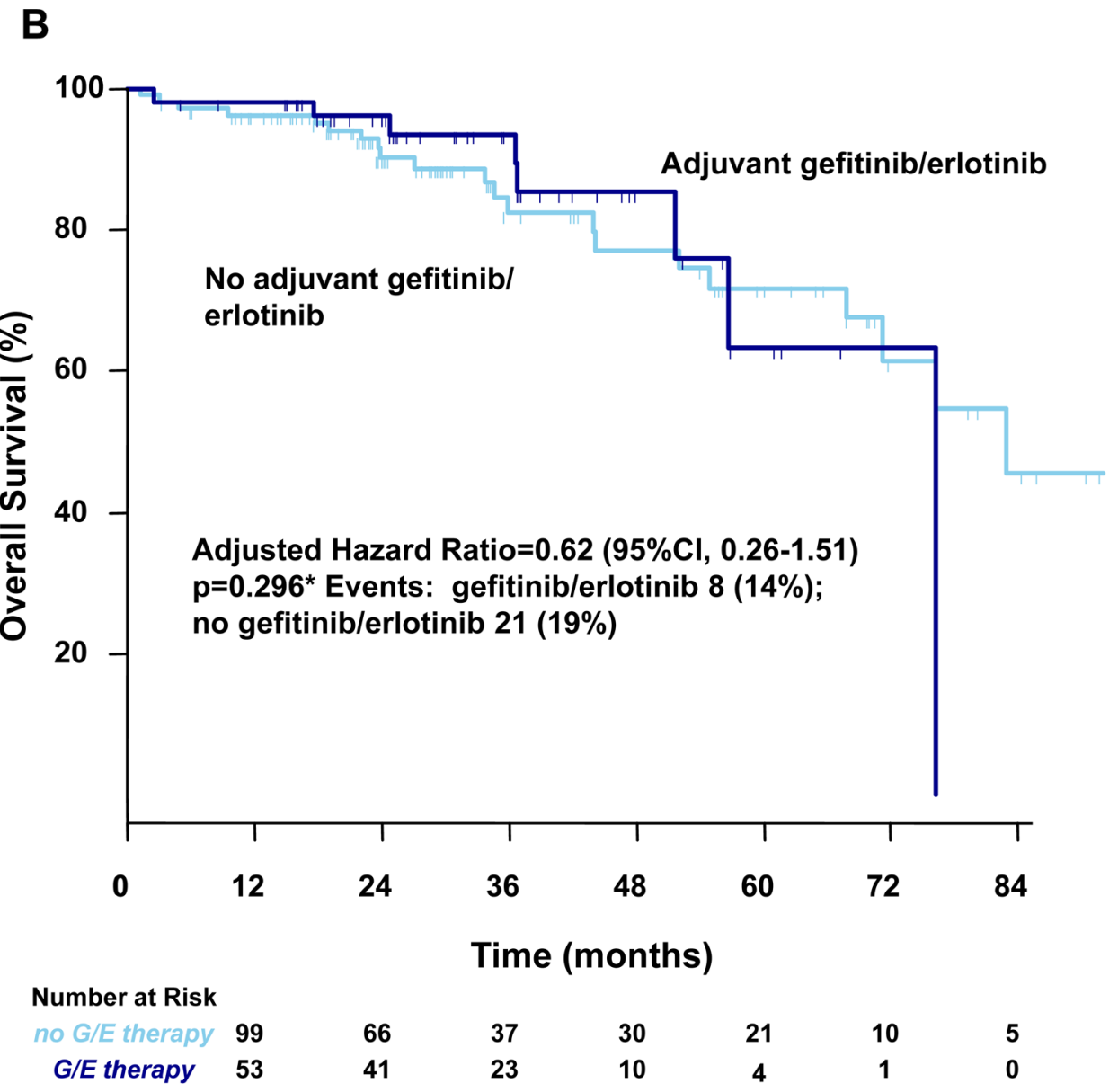

Figure 1. Kaplan-Meier Curves for Survival

Kaplan-Meier curves for Disease Free Survival (Panel A) and for Overall Survival (Panel B) by gefitinib/erlotinib therapy. G/E denotes gefitinib/erlotinib. *Hazard ratios were calculated with the use of a Cox proportional-hazards model, with the type of surgery, stage, cisplatin chemotherapy, sex as covariates and gefitinib/erlotinib therapy as time-dependent factor; ${ }^{\dagger}$ Cox proportional-hazards model with gefitinib/erlotinib therapy as time-dependent factor. With respect to the disease free survival and overall survival, results of a log-rank test, $\mathrm{p}=0.122$ (Panel A) and $\mathrm{p}=0.912$ (Panel B). 
-Resected Stage I-IIIA lung adenocarcinoma

-Activating EGFR mutation

(exon 19 deletion or

exon 21 L858R)

-Perioperative cytotoxic

chemotherapy as indicated $\mathrm{N}=320$

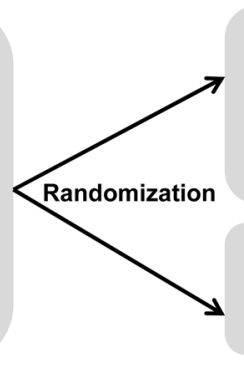

Adjuvant erlotinib or gefitinib

for up 2 years and routine surveillance $\mathrm{N}=160$

\section{Routine} surveillance $\mathrm{N}=160$
- Continued surveillance -All patients with recurrence during surveillance will receive first line EGFR TKI and will be assessed for radiographic response, progression free and overall survival

-Patients with recurrence after adjuvant TKI should have tumor biopsy to compare molecular characteristics with resected tumor

Figure 2.

Schema for a clinical trial of adjuvant erlotinib or gefitinib. EGFR denotes epidermal growth factor receptor. Primary endpoint is disease-free survival proportion at 2 years from time of randomization. 
Table 1

Patient demographic and baseline characteristics.

\begin{tabular}{|llll|}
\hline Characteristic & $\begin{array}{l}\text { Adjuvant erlotinib/gefitinib } \\
\text { N=56 }\end{array}$ & $\begin{array}{l}\text { No adjuvant erlotinib/gefitinib } \\
\text { N=111 }\end{array}$ & p-value \\
\hline Age -years & & & $.050^{\dagger}$ \\
Median & 66 & 70 & \\
Range & 37 to 88 & 35 to 89 & 1.00 \\
\hline Sex-- no. (\%) & & & \\
Male & $15(27)$ & $31(28)$ & .002 \\
Female & $41(73)$ & $80(72)$ & \\
\hline Pathologic stage-no. (\%) & & & \\
I & $30(54)$ & $87(78)$ & 0.016 \\
II & $11(20)$ & $14(13)$ & 0.010 \\
III & $15(27)$ & $10(9)$ & \\
\hline Cisplatin chemotherapy-no. $(\%)$ & $22(39)$ & $23(21)$ & \\
\hline Surgery Type-no. (\%) & & & \\
Bilobectomy & $3(5)$ & $91(82)$ & \\
Lobectomy & $49(88)$ & 0 & \\
Pneumonectomy & $1(2)$ & $11(10)$ & \\
Segmentectomy & $2(4)$ & & \\
Wedge & & $912)$ & \\
\hline
\end{tabular}

p-value calculated using Wilcoxon rank-sum test. Other p-values calculated using Fisher exact test;

Percentages may not sum to 100 because of rounding. 
Table 2

Characteristics of patients who recurred or died

\begin{tabular}{|lll|}
\hline Characteristic & $\begin{array}{l}\text { Adjuvant erlotinib/gefitinib } \\
\mathbf{N = 1 3}\end{array}$ & $\begin{array}{l}\text { No adjuvant erlotinib/gefitinib } \\
\mathbf{N = 4 3}\end{array}$ \\
\hline Age -years & & \\
Median & 62 & 68 \\
Range & 38 to 88 & 35 to 85 \\
\hline Sex-- no. (\%) & & \\
Male & & $13(30)$ \\
Female & $6(46)$ & $30(70)$ \\
\hline Pathologic stage-no. (\%) & $7(54)$ & \\
I & & $30(70)$ \\
II & $3(23)$ & $5(12)$ \\
III & $4(31)$ & $8(19)$ \\
Cisplatin chemotherapy-no. (\%) & $7(54)$ & $10(23)$ \\
\hline
\end{tabular}

Percentages may not sum to 100 because of rounding. 
Table 3

Multivariate Disease Free Survival Analysis

\begin{tabular}{|c|c|c|c|c|}
\hline$n=167$ & $\mathbf{N}($ event $\mathbf{N})$ & 2 -year survival (95\% CI) & Adjusted Hazard Ratio ${ }^{*}(95 \%$ CI $)$ & Adjusted $p$-value \\
\hline Adjuvant Erlotinib/Gefitinib & $56(13)$ & $89 \%$ (77 to 95$)$ & $0.53(0.28$ to 1.03$)$ & 0.06 \\
\hline No Adjuvant Erlotinib/Gefitinib & $111(43)$ & $72 \%$ (61 to 80$)$ & & \\
\hline
\end{tabular}

* adjusted for sex, type of surgery, stage and adjuvant cisplatin chemotherapy; Hazard Ratio <1.00 indicates improved survival; $95 \%$ CI denotes 95\% confidence interval 
Table 4

Multivariate Overall Survival Analysis

\begin{tabular}{|lllll|}
\hline $\mathbf{n = 1 6 7}$ & $\mathbf{N}($ event $\mathbf{N})$ & $\mathbf{2}$-year survival (95\% CI) & Adjusted Hazard Ratio ${ }^{\text {(95\%) }}$ CI & Adjusted $\boldsymbol{p}$-value \\
\hline Adjuvant Erlotinib/Gefitinib & $56(8)$ & $96 \%(85$ to 99) & $0.62(0.26$ to 1.51$)$ & 0.296 \\
\hline No Adjuvant Erlotinib/Gefitinib & $111(21)$ & $90 \%(82$ to 95$)$ & & \\
\hline
\end{tabular}

adjusted for sex, type of surgery, stage and adjuvant cisplatin chemotherapy; Hazard Ratio <1.00 indicates improved survival; 95\% CI denotes $95 \%$ confidence interval 